\title{
THE DYNAMICAL ANDRÉ-OORT CONJECTURE: UNICRITICAL POLYNOMIALS
}

\author{
D. GHIOCA, H. KRIEGER, K. NGUYEN, AND H. YE
}

\begin{abstract}
We establish the equidistribution with respect to the bifurcation measure of postcritically finite maps in any one-dimensional algebraic family of unicritical polynomials. Using this equidistribution result, together with a combinatorial analysis of certain algebraic correspondences on the complement of the Mandelbrot set $\mathcal{M}_{2}$ (or generalized Mandelbrot set $\mathcal{M}_{d}$ for degree $d>2$ ), we classify all algebraic curves $C \subset \mathbb{C}^{2}$ with Zariski-dense subsets of points $(a, b) \in C$, such that both $z^{d}+a$ and $z^{d}+b$ are simultaneously postcritically finite for a fixed degree $d \geq 2$. Our result is analogous to the famous result of André And98 regarding plane curves which contain infinitely many points with both coordinates CM parameters, and is the first complete case of the dynamical André-Oort phenomenon studied by Baker and DeMarco BD11.
\end{abstract}

\section{INTRODUCTION}

In the past 20 years there was a considerable interest in studying the principle of unlikely intersections in arithmetic geometry (for a comprehensive discussion, see the book of Zannier [Zan12]). Informally, this principle of unlikely intersections (of which special cases are both the André-Oort and the Pink-Zilber conjectures) predicts that each time an intersection of an algebraic variety with a family of algebraic varieties is larger than expected, then this is explained by the presence of a rigid geometric constraint. Motivated by a version of the Pink-Zilber Conjecture for semiabelian schemes, Masser and Zannier (see [MZ10, MZ12]) proved that in a non-constant elliptic family $E_{t}$ parametrized by $t \in \mathbb{C}$, for any two sections $\left\{P_{t}\right\}_{t}$ and $\left\{Q_{t}\right\}_{t}$, if there exist infinitely many $t \in \mathbb{C}$ such that both $P_{t}$ and $Q_{t}$ are torsion points on $E_{t}$, then the two sections are linearly dependent.

Motivated by a question of Zannier, Baker and DeMarco [BD11] proved a first result for the unlikely intersections principle in the context of algebraic dynamics. More precisely, Baker and DeMarco showed that for an integer $d \geq 2$, and for two complex numbers $a$ and $b$, if there exist infinitely many $t \in \mathbb{C}$ such that both $a$ and $b$ are preperiodic under the action of $z \mapsto z^{d}+t$, then $a^{d}=b^{d}$. Baker and DeMarco's result BD11] can be seen as an analogue of Masser and Zannier result [MZ10, MZ12] (which can be reformulated for simultaneous preperiodic points in a family of Lattés maps) without the presence of an algebraic group. The absence of an algebraic group in the background is an added difficulty for the problem, which is solved by Baker and DeMarco employing an argument which relies on a theorem regarding the equidistribution of points of small height for algebraic dynamical systems (see [BR06, CL06, FRL06]). New results

2010 Mathematics Subject Classification. Primary 37F50; Secondary 37F05.

Key words and phrases. Mandelbrot set, unlikely intersections in dynamics.

The research of H.K. was partially supported by an NSF grant, while the research of D.G., K.N. and H.Y. was partially supported by NSERC grants. 
followed (see [GHT12, BD13]) extending the results of [BD11] to arbitrary 1-parameter families of polynomials.

In [BD13, Baker and DeMarco posed a very general question for families of dynamical systems, which is motivated by the classical André-Oort. As a parallel to the classical André-Oort Conjecture, Baker and DeMarco's question asks that if a subvariety $V$ of the moduli space of rational maps of given degree contains a Zariski dense set of special points, then $V$ itself is special (i.e., cut out by critical orbit relations; see [BD13] for more details). The special points of the moduli space in Baker-DeMarco's question are the ones corresponding to postcritically finite (PCF) maps $f$, i.e. each critical point of $f$ is preperiodic. In this article we prove a general result for curves supporting this Dynamical André-Oort Conjecture.

Theorem 1.1. Let $C$ be an irreducible algebraic plane curve defined over $\mathbb{C}$, and let $d \geq 2$ be an integer. There exist infinitely many points $(a, b) \in C$, such that both $z \mapsto z^{d}+a$ and $z \mapsto z^{d}+b$ are postcritically finite, if and only if one of the following conditions holds:

(1) there exists $t_{0} \in \mathbb{C}$ such that $z \mapsto z^{d}+t_{0}$ is $P C F$ and $C$ is the curve $\left\{t_{0}\right\} \times \mathbb{A}^{1}$;

(2) there exists $t_{0} \in \mathbb{C}$ such that $z \mapsto z^{d}+t_{0}$ is $P C F$ and $C$ is the curve $\mathbb{A}^{1} \times\left\{t_{0}\right\}$;

(3) there exists a $(d-1)$-st root of unity $\zeta$ such that $C$ is the zero locus of the equation $y-\zeta x=0$.

In GKN, Theorem 1.1 was proven in the special case $C$ is the graph of a polynomial. The extension to arbitrary curves in Theorem 1.1 requires overcoming several technical difficulties. We also note that Theorem 1.1 can be viewed as a dynamical analogue of André's theorem And98. regarding plane curves containing infinitely many points with both coordinates CM points in the parameter space of elliptic curves. In the world of polynomial dynamics, the equivalent notion of a CM elliptic curve is a PCF polynomial. Indeed, the parallel between the two can be viewed also at the level of arboreal Galois representation associated to a polynomial which is expected to have smaller image for PCF maps, analogous to the situation for the Galois representation associated to an elliptic curve, which has smaller image in the case of CM elliptic curves; for more details, see [Jon13, Pina, Pinb, Pinc].

We observe that it is immediate to see that a curve of the form (1) to (3) as in the conclusion of Theorem 1.1 contains infinitely many points with both coordinates PCF parameters; the difficulty in Theorem 1.1 is proving that only such curves have infinitely many such points. If $C$ does not project dominantly onto one of the axis of $\mathbb{A}^{2}$, it is immediate to see that $C$ must have the form (1) or (2) above. So, the content of Theorem 1.1 is to show that when $C$ projects dominantly onto both axis of $\mathbb{A}^{2}$, and in addition $C$ contains infinitely many points with both coordinates PCF parameters, then $C$ must be of the form (3) as in the conclusion of our result.

Theorem 1.1 can be viewed as a generalization of the problem studied in [BD11, as follows. Given a plane curve $C$, we have two families of polynomials parametrized by the points $t \in C$ : $\mathbf{f}_{1, t}(z):=z^{d}+\pi_{1}(t)$ and $\mathbf{f}_{2, t}(z):=z^{d}+\pi_{2}(t)$, where $\pi_{1}$ and $\pi_{2}$ are the two projections of $C$ onto the two coordinate axis of the affine plane. Then we study under what conditions there are infinitely many $t \in C$ such that 0 is preperiodic under both $\mathbf{f}_{1, t}$ and $\mathbf{f}_{2, t}$. More generally, one could consider any two families of rational maps $\mathbf{f}_{1, t}$ and $\mathbf{f}_{2, t}$ parametrized by points $t$ on some curve $C$, take any two rational maps $c_{1}, c_{2}: C \longrightarrow \mathbb{P}^{1}$, and ask under what conditions on the curve $C$, on the two families $\mathbf{f}_{1}$ and $\mathbf{f}_{2}$, and on the starting points $c_{1}$ and $c_{2}$, there exist infinitely many points $t \in C$ such that both $c_{1}(t)$ and $c_{2}(t)$ are preperiodic under the action of $\mathbf{f}_{1, t}$, respectively of $\mathbf{f}_{2, t}$. However 
this is a very hard question, and there are only a handful of results with restricted conditions in the literature; see [BD11, GHT12, BD13, GHT15]. For examples in [BD11, GHT12, BD13, $\mathbf{f}_{1}$ and $\mathbf{f}_{2}$ are families of polynomials and $C=\mathbb{A}^{1}$. In GHT15, the family of rational functions, parametrized by a projective curve $C$, must have exactly one degenerate point on $C$ and also the families $\mathbf{f}_{1}$ and $\mathbf{f}_{2}$ must satisfy additional technical conditions. In this article, we release all the restrictions on the curve $C$, which parametrizes a family of unicritical polynomials. Also, we note that the result of GKN] relied on the results from GHT12, hence the restriction to curves $C$ which were graphs of polynomials because then the families of maps $\mathbf{f}_{1, t}$ and $\mathbf{f}_{2, t}$ were parametrized by the affine line.

One of the main ingredients of our article (and also of all of the above articles) is the arithmetic equidistribution of small points on an algebraic variety (in the case of $\mathbb{P}^{1}$, see [BR06, FRL06, in the general case of curves, see [CL06], while for arbitrary varieties, see [Yua08]). Another main ingredient of this article is the geometric properties of the generalized Mandelbrot sets $\mathcal{M}_{d}$ (recall that $\mathcal{M}_{d}$ is the set of all $t \in \mathbb{C}$ where the orbit of 0 under $z \mapsto z^{d}+t$ is bounded). More precisely, we use the combinatorial behaviour of the landing of the external rays, from which we get the precise equations for the curves $C$ in Theorem 1.1. Using Yuan's powerful theorem Yua08, we show that postcritically finite maps equidistribute on the parameter space with respect to the bifurcation measure; see Theorem 3.1. Assuming there exist infinitely many points $(a, b)$ on the plane curve $C$ such that both $z^{d}+a$ and $z^{d}+b$ are PCF, then the potential (escape-rate) functions for the bifurcation measures (with respect to the families $z^{d}+\pi_{1}(t)$ and $z^{d}+\pi_{2}(t)$, where $\pi_{1}$ and $\pi_{2}$ are the two projections of $C$ on the coordinates of $\mathbb{A}^{2}$ ) are proportional to each other; see Theorem 4.1. Hence we get an algebraic correspondence on the $d$-th generalized Mandelbrot set $\mathcal{M}_{d}$ : for each $(a, b) \in C$, we have that $a \in \mathcal{M}_{d}$ if and only if $b \in \mathcal{M}_{d}$. Using the theory of landing external rays on the $d$-th generalized Mandelbrot set we prove that the only algebraic correspondences on $\mathcal{M}_{d}$ are linear given by an equation as in the conclusion of Theorem 1.1 .

We are indebted to Laura DeMarco and Thomas Tucker for their careful reading of, and helpful comments on, an early version of this article. We also thank Bjorn Poonen and Curt McMullen for helpful discussions during the writing of this paper.

\section{Preliminaries}

In this section, we introduce terminologies and results (e.g. Yuan's arithmetic equidistribution theorem Yua08]) as needed for the latter sections. Though Yuan's equidistribution works for varieties of all dimensions, we focus on the one dimensional case.

2.1. The height functions. Let $K$ be a number field and $\bar{K}$ be the algebraic closure of $K$. The number field $K$ is naturally equipped with a set $\Omega_{K}$ of pairwise inequivalent nontrivial absolute values, together with positive integers $N_{v}$ for each $v \in \Omega_{K}$ such that

- for each $\alpha \in K^{*}$, we have $|\alpha|_{v}=1$ for all but finitely many places $v \in \Omega_{K}$.

- every $\alpha \in K^{*}$ satisfies the product formula

$$
\prod_{v \in \Omega_{K}}|\alpha|_{v}^{N_{v}}=1
$$

For each $v \in \Omega_{K}$, let $K_{v}$ be the completion of $K$ at $v$, let $\bar{K}_{v}$ be the algebraic closure of $K_{v}$ and let $\mathbb{C}_{v}$ denote the completion of $\bar{K}_{v}$. We fix an embedding of $\bar{K}$ into $\mathbb{C}_{v}$ for each $v \in \Omega_{K}$; hence 
we have a fixed extension of $|\cdot|_{v}$ on $\bar{K}$. When $v$ is archimedean, then $\mathbb{C}_{v} \cong \mathbb{C}$. For any $x \in \bar{K}$, the Weil height is

$$
h(x)=\frac{1}{[K(x): K]} \sum_{y \in \operatorname{Gal}(\bar{K} / K) \cdot x} \sum_{v \in \Omega_{K}} \log ^{+}|y|_{v}
$$

where $\log ^{+} z=\log \max \{1, z\}$ for any real number $z$.

Let $f \in K[z]$ be any polynomial with degree $d \geq 2$. We use the notation $f^{n}$ for the composition of $f$ with itself $n$ times. As introduced by Call and Silverman [CS93, we have the following canonical height for every $x \in \bar{K}$

$$
\hat{h}_{f}(x)=\lim _{n \rightarrow \infty} \frac{h\left(f^{n}(x)\right)}{d^{n}}
$$

where $h(x)$ is the Weil height from 2.2 . Call and Silverman CS93] showed that the above canonical height is well-defined, and moreover, $\hat{h}_{f}(x) \geq 0$ with equality if and only if $x$ is preperiodic under the iteration of $f$. Hence, $f$ is postcritically finite if and only if all its critical points have canonical height zero.

2.2. Adelic metrized line bundle and equidistribution. Let $\mathcal{L}$ be a line bundle of a nonsingular projective curve $X$ over a number field $K$. As in Subsection 2.1, $K$ is naturally equipped with absolutes $|\cdot|_{v}$ for $v \in \Omega_{K}$. A metric $\|\cdot\|_{v}$ on $\mathcal{L}$ is a collection of norms, one for each $x \in X\left(K_{v}\right)$, on the fibres $\mathcal{L}(x)$ of the line bundle, with

$$
\|\alpha s(x)\|_{v}=|\alpha|_{v}\|s(x)\|_{v}
$$

for any section $s$ of $\mathcal{L}$. An adelic metrized line bundle $\overline{\mathcal{L}}=\left\{\mathcal{L},\left\{\|\cdot\|_{v}\right\}_{v \in \Omega_{K}}\right\}$ over $\mathcal{L}$ is a collection of metrics on $\mathcal{L}$, one for each place $v \in \Omega_{K}$, satisfying certain continuity and coherence conditions; see [ha95a, Zha95b].

For example, we can define adelic metrized line bundles for $\mathbb{P}^{1}$ over the line bundle $\mathcal{L}=\mathcal{O}_{\mathbb{P}^{1}}(1)$. Let $s=u_{0} X_{0}+u_{1} X_{1}$ be a global section of $\mathcal{L}=\mathcal{O}_{\mathbb{P}^{1}}(1)$, where $u_{0}$ and $u_{1}$ are scalars. The metrics are defined for each $\left[x_{0}: x_{1}\right] \in \mathbb{P}^{1}(\bar{K})$ as

$$
\left\|s\left(\left[x_{0}: x_{1}\right]\right)\right\|_{v}:=\frac{\left|u_{0} x_{0}+u_{1} x_{1}\right|_{v}}{\max \left\{\left|x_{0}\right|_{v},\left|x_{1}\right|_{v}\right\}}
$$

for places $v \in \Omega_{K}$. It can be checked without any difficulty that $\overline{\mathcal{L}}:=\left\{\mathcal{L},\left\{\|\cdot\|_{v}\right\}_{v \in \Omega_{K}}\right\}$ defined this way is an adelic metrized line bundle over $\mathcal{L}$. Moreover, we can work with pullback metrics by an endomorphism of $\mathbb{P}^{1}$. More precisely, let $F\left(\left[x_{0}: x_{1}\right]\right)=\left(F_{0}\left(x_{0}, x_{1}\right): F_{1}\left(x_{0}, x_{1}\right)\right)$ be an endomorphism of $\mathbb{P}^{1}$ where $F_{1}$ and $F_{2}$ are coprime homogeneous polynomials of degree $d$. The metrics on $s=u_{0} X_{0}+u_{1} X_{1}$ are defined as

$$
\left\|s\left(\left[x_{0}: x_{1}\right]\right)\right\|_{v}^{F}:=\frac{\left|u_{0} x_{0}+u_{1} x_{1}\right|_{v}}{\max \left\{\left|F_{0}\left(x_{0}, x_{1}\right)\right|_{v},\left|F_{1}\left(x_{0}, x_{1}\right)\right|_{v}\right\}^{1 / d}}
$$

Hence $\overline{\mathcal{L}}_{F}:=\left\{\mathcal{L},\left\{\|\cdot\|_{v}^{F}\right\}_{v \in \Omega_{K}}\right\}$ is an adelic metrized line bundle over $\mathcal{L}$.

A sequence $\left\{\mathcal{L},\left\{\|\cdot\|_{v, n}\right\}_{v \in \Omega_{K}}\right\}_{n \geq 1}$ of adelic metrized line bundles over $\mathcal{L}$ is convergent to $\left\{\mathcal{L},\left\{\|\cdot\|_{v}\right\}_{v \in \Omega_{K}}\right\}$, if for all $n$ and all but finitely many $v \in \Omega_{K},\|\cdot\|_{v, n}=\|\cdot\|_{v}$, and if $\left\{\log \frac{\|\cdot\|_{v, n}}{\|\cdot\|_{v}}\right\}_{n \geq 1}$ converges to 0 uniformly on $X(K)$ for all $v \in \Omega_{K}$. It is clear that the limit $\left\{\mathcal{L},\left\{\|\cdot\|_{v}\right\}_{v \in \Omega_{K}}\right\}$ is an adelic metrized line bundle. 
All metrics we consider here are induced by models or uniform limits of metrics from models; see[Zha95b, Yua08]. An adelic metrized line bundle $\overline{\mathcal{L}}$ is algebraic if there is a model $\mathcal{X}$ of $X$ that induces the metrics on $\mathcal{L}$. An algebraic adelic metrized line bundle $\overline{\mathcal{L}}$ is semipositive if $\overline{\mathcal{L}}$ has semipositive curvatures at archimedean places and non-negative degree on any complete vertical curve of $\mathcal{X}$. An adelic metrized line bundle $\overline{\mathcal{L}}$ is semipositive if it is the uniform limit of a sequence of algebraic adelic semipositive metrics over $\mathcal{L}$.

For a semipositive line bundle $\overline{\mathcal{L}}$, we can define a height for each subvariety $Y$ of $X$ (denoted $\left.\hat{h}_{\overline{\mathcal{L}}}(Y)\right)$; see [Zha95b] for more details. Let $X$ be a nonsingular projective curve. In the case of points on $X$, the height for $x \in X(\bar{K})$ is given by

$$
\hat{h}_{\overline{\mathcal{L}}}(x)=\frac{1}{|\operatorname{Gal}(\bar{K} / K) \cdot x|} \sum_{y \in \operatorname{Gal}(\bar{K} / K) \cdot x} \sum_{v \in \Omega_{K}}-N_{v} \log \|s(y)\|_{v}
$$

where $|\operatorname{Gal}(\bar{K} / K) \cdot x|$ is the number of points in the Galois orbits of $x$, and $s$ is any meromorphic section of $\mathcal{L}$ with support disjoint from $\operatorname{Gal}(\bar{K} / K) \cdot x$. A sequence of points $x_{n} \in X(\bar{K})$ is small, if $\lim _{n \rightarrow \infty} \hat{h}_{\overline{\mathcal{L}}}\left(x_{n}\right)=\hat{h}_{\overline{\mathcal{L}}}(X)$.

Theorem 2.1. YYua08, Theorem 3.1] Suppose $X$ is a projective curve over a number field $K$, and $\overline{\mathcal{L}}$ is a metrized line bundle over $X$ such that $\mathcal{L}$ is ample and the metric is semipositive. Let $\left\{x_{n}\right\}$ be a non-repeating sequence of points in $X(\bar{K})$ which is small. Then for any $v \in \Omega_{K}$, the Galois orbits of the sequence $\left\{x_{n}\right\}$ are equidistributed in the analytic space $X_{\mathbb{C}_{v}}^{\text {an }}$ with respect to the probability measure $d \mu_{v}=c_{1}(\overline{\mathcal{L}})_{v} / \operatorname{deg}_{\mathcal{L}}(X)$.

Remark 1. When $v$ is archimedean, $X_{\mathbb{C}_{v}}^{a n}$ corresponds to $X(\mathbb{C})$ and the curvature $c_{1}(\overline{\mathcal{L}})_{v}$ of the metric $\|\cdot\|_{v}$ is given by $c_{1}(\overline{\mathcal{L}})_{v}=\frac{\partial \bar{\partial}}{\pi i} \log \|\cdot\|_{v}$. For non-archimedean place $v, X_{\mathbb{C}_{v}}^{a n}$ is the Berkovich space associated to $X\left(\mathbb{C}_{v}\right)$, and Chambert-Loir [CL06] constructed an analog of curvature on $X_{\mathbb{C}_{v}}^{a n}$. The precise meaning of the equidistribution above is:

$$
\lim _{n \rightarrow \infty} \frac{1}{\left|\operatorname{Gal}(\bar{K} / K) \cdot x_{n}\right|} \sum_{y \in \operatorname{Gal}(\bar{K} / K) \cdot x_{n}} \delta_{y}=\mu_{v}
$$

where $\delta_{y}$ is point mass probability measure supported on $y \in X_{\mathbb{C}_{v}}^{a n}$, and the limit is the weak limit for probability measures on compact space $X_{\mathbb{C}_{v}}^{a n}$.

\section{Equidistribution of PCF points}

In Sections 3 and 4, we prove that for a (one dimensional and non-isotrivial) family of unicritical polynomials with degree $d \geq 2$, the set of postcritically finite polynomials equidistributes on the parameter space. The main tool we use in this section is the arithmetic equidistribution theorem introduced in the previous section; for the setup of the equidistribution theorem, we follow GHT15]. We start by stating Theorem 3.1 which is our main goal; in order to do this we need to set up the proper notation.

3.1. Statement of the equidistribution theorem for PCF parameters. For the definition of algebraic families of unicritical polynomials, we follow [Dem]. Let $f: X^{\prime} \times \mathbb{C} \rightarrow \mathbb{C}$ be a one dimensional algebraic family of unicritical polynomials of degree $d \geq 2$. That is, $X^{\prime}$ is a Zariski dense, open subset of an irreducible, smooth curve $X$ defined over $\mathbb{C}$, while $\psi: X^{\prime} \longrightarrow \mathbb{A}^{1}$ is a morphism, and $f$ is a polynomial map of degree $d$ given by $f_{\psi(t)}(z):=f(t, z)=z^{d}+\psi(t)$, for each 
$t \in X^{\prime}(\mathbb{C})$. We say that $f$ is isotrivial if $\psi$ is a constant map. Since there is nothing to study for an isotrivial family of unicritical polynomials, we focus on the non-isotrivial case. In addition, we assume $X$ and $X^{\prime}$ are defined over a number field $K$. If $\psi$ is a morphism defined over $K$, then we call $f$ an algebraic family of unicritical polynomials over the number field $K$. We can view $X$ as a parameter space for an algebraic family of unicritical polynomials. The main goal for us is proving the following result.

Theorem 3.1. Let $f: X^{\prime} \times \mathbb{C} \rightarrow \mathbb{C}$ be a non-isotrivial, one dimensional algebraic family of degree $d \geq 2$ unicritical polynomials over a number field $K$. The set of parameters $t \in X^{\prime}(\bar{K})$, for which $f(t, z): \mathbb{C} \rightarrow \mathbb{C}$ is postcritically finite, equidistributes on the parameter space $X(\mathbb{C})$ (with respect to the normalized bifurcation measure).

We postpone the proof of this theorem to Subsection 4.3 (for a precise definition of the equidistribution, see Remark 1). In Subsection 4.1 we define the (normalized) biffurcation measure and also show its connection with the measures corresponding to certain adelic metrized line bundles as appearing in the work of Yuan Yua08. The key to our proof of Theorem 3.1 is the equidistribution theorem of Yuan (see Theorem 2.1 and its consequence to our setting stated in Theorem 3.4.

3.2. Metrics on a line bundle. As previously stated, a non-isotrivial, one dimensional algebraic family of unicritical polynomials over a number field $K$ is uniquely determined by a morphism $\psi: X^{\prime} \rightarrow \mathbb{A}^{1}$, where $X^{\prime}$ is a Zariski dense open subset of an irreducible, nonsingular projective curve $X$ defined over $K$. Hence the morphism $\psi: X^{\prime} \longrightarrow \mathbb{A}^{1}$ induces a unique morphism $\psi: X \longrightarrow \mathbb{P}^{1}$ (for the sake of simplifying the notation, we use the same notation for both morphisms).

Let $\mathcal{L}$ be the line bundle on the projective curve $X$ which is the pullback of $\mathcal{O}_{\mathbb{P}^{1}}(1)$ by $\psi$, i.e. $\mathcal{L}:=\psi^{*} \mathcal{O}_{\mathbb{P}^{1}}(1)$. Next we are going to introduce metrics on this line bundle. Let $S$ be the set of poles of $\psi$ on $X$, i.e. $S$ consists of all $x \in X$ such that $\psi(x)=[1: 0]$ (the infinity point of $\mathbb{P}^{1}$ ). Let $X_{0}, X_{1}$ be the canonical sections on $\mathbb{P}^{1}$, and $s:=\psi^{*}\left(u_{0} X_{0}+u_{1} X_{1}\right)$ be a section of the line bundle $L$ with $u_{0}$ and $u_{1}$ being the scalars. For any point $t \in X\left(\mathbb{C}_{v}\right) \backslash S$, we define the metrics for each $n \geq 1$ and each place $v \in \Omega_{K}$ as follows:

$$
\|s(t)\|_{v, n}:=\frac{\left|u_{0} \psi(t)+u_{1}\right|_{v}^{1 / d_{\psi}}}{\max \left\{1,\left|f_{\psi(t)}^{n}(0)\right|_{v}\right\}^{1 /\left(d_{\psi} \cdot d^{n-1}\right)}}
$$

where $d_{\psi}$ is the degree of the morphism $\psi: X \rightarrow \mathbb{P}^{1}$. Moreover, for each $t_{0} \in S \subset X\left(\mathbb{C}_{v}\right)$, we define

$$
\left\|s\left(t_{0}\right)\right\|_{v, n}:=v-\lim _{t \rightarrow t_{0}}\|s(t)\|_{v, n}=\left|u_{0}\right|_{v}^{1 / d_{\psi}}
$$

The last equality in the above formula is obvious once we notice that when $t$ is close to $t_{0}$, we have $\left|f_{\psi(t)}^{n}(0)\right|_{v}^{1 / d^{n-1}} \sim|\psi(t)|_{v}$.

Lemma 3.2. For any nonarchimedean place $v \in \Omega_{K}$ and any integer $n \geq 1$, we have

$$
\|\cdot\|_{v, n}=\|\cdot\|_{v, 1}
$$

on the line bundle $\mathcal{L}$. 
Proof. It suffices to show that $\max \left\{1,\left|f_{\psi(t)}^{n}(0)\right|_{v}\right\}=\max \left\{1,|\psi(t)|_{v}^{d^{n-1}}\right\}$ for all $t \in X\left(\mathbb{C}_{v}\right) \backslash S$, where $S$ is the set of poles for $\psi$. We prove it by induction. Suppose $\max \left\{1,\left|f_{\psi(t)}^{n}(0)\right|_{v}\right\}=$ $\max \left\{1,|\psi(t)|_{v}^{d^{n-1}}\right\}$. If $|\psi(t)|_{v} \leq 1$, then $\left|f_{\psi(t)}^{n}(0)\right|_{v} \leq 1$. Hence $\left|f_{\psi(t)}^{n+1}(0)\right|_{v}=\left|\left(f_{\psi(t)}^{n}(0)\right)^{d}+\psi(t)\right|_{v} \leq$ $\max \left\{\left|\left(f_{\psi(t)}^{n}(0)\right)^{d}\right|_{v},|\psi(t)|_{v}\right\} \leq 1$ as $v$ is nonarchimedean. Otherwise if $|\psi(t)|_{v}>1$ and $\left|f_{\psi(t)}^{n}(0)\right|_{v}=$ $|\psi(t)|_{v}^{d^{n-1}} \geq|\psi(t)|_{v}>1$, then $\left|f_{\psi(t)}^{n+1}(0)\right|_{v}=\left|\left(f_{\psi(t)}^{n}(0)\right)^{d}+\psi(t)\right|_{v}=\left|\left(f_{\psi(t)}^{n}(0)\right)^{d}\right|_{v}=|\psi(t)|_{v}^{d^{n}}$.

We define the metric

$$
\|s(t)\|_{v}:=\lim _{n \rightarrow \infty}\|s(t)\|_{v, n} \text { for each place } v
$$

and we prove next that $\log \|\cdot\|_{v, n}$ converges uniformly to $\log \|\cdot\|_{v}$.

Proposition 3.3. For each place $v \in \Omega_{K}, \log \|\cdot\|_{v, n}$ converges uniformly on $X\left(\mathbb{C}_{v}\right)$ to $\log \|\cdot\|_{v}$.

Proof. Fix a place $v \in \Omega_{K}$ and a real number $R$ greater than 3 . Let

$$
R_{v, n}(t):=\max \left\{1,\left|f_{\psi(t)}^{n}(0)\right|_{v}\right\}^{1 / d^{n-1}} .
$$

To prove this proposition, it suffices to show that $\left\{\log R_{v, n}(t)\right\}_{n \geq 1}$, as a sequence of functions on $X\left(\mathbb{C}_{v}\right)$, converges uniformly.

First we prove the uniform convergence assuming $|\psi(t)|_{v} \leq R$. Then from the definition of $R_{v, n}(t)$, we know that

$$
\begin{aligned}
R_{v, n+1}^{d^{n}}(t) & \leq\left(R_{v, n}^{d^{n-1}}(t)\right)^{d}+R \\
& \leq(R+1) \cdot R_{v, n}^{d^{n-1}}(t), \text { since } R_{v, n}(t) \geq 1 \\
& \leq 2 R \cdot R_{v, n}^{d^{n}}(t)
\end{aligned}
$$

Similarly, if $R_{v, n}^{d^{n}}(t) \geq 2 R$, then $R_{v, n+1}^{d^{n}}(t) \geq\left(R_{v, n}^{d^{n-1}}(t)\right)^{d}-R \geq R_{v, n}^{d^{n}}(t) / 2 \geq R_{v, n}^{d^{n}}(t) / 2 R$. On the other hand, if $R_{v, n}^{d^{n}}(t)<2 R$, then $R_{v, n+1}^{d^{n}}(t) \geq R_{v, n}^{d^{n}}(t) / 2 R$. So in all cases,

$$
\frac{1}{(2 R)^{1 / d^{n-1}}} \leq \frac{R_{v, n+1}(t)}{R_{v, n}(t)} \leq(2 R)^{1 / d^{n-1}}
$$

which yields the uniform convergence of $\left\{\log R_{v, n}(t)\right\}_{n}$ (by taking logarithms in (3.3) and the use a telescoping sum) for all $t \in X\left(\mathbb{C}_{v}\right)$ satisfying $|\psi(t)|_{v} \leq R$.

Secondly, we assume $t \in X\left(\mathbb{C}_{v}\right) \backslash S$ such that $|\psi(t)|_{v}>R$. We prove by induction on $n$ that $R_{v, n}^{d^{n-1}}(t) \geq|\psi(t)|_{v}$. Indeed, the case $n=1$ is obvious, while in general (also noting that $R>3$ and $d \geq 2$ ) we have:

$$
R_{v, n+1}^{d^{n}}(t) \geq\left(R_{v, n}^{d^{n-1}}(t)\right)^{d}-|\psi(t)|_{v} \geq R_{v, n}^{d^{n}}(t)-\frac{R_{v, n}^{d^{n}}(t)}{2} \geq \frac{R_{v, n}^{d^{n}}(t)}{2} \geq R_{v, n}^{d^{n-1}}(t) \geq|\psi(t)|_{v} .
$$

Then it is easy to see that

$$
\left|R_{v, n+1}^{d^{n}}(t)-\left(R_{v, n}^{d^{n-1}}(t)\right)^{d}\right| \leq|\psi(t)|_{v} \leq \frac{R_{v, n}^{d^{n}}(t)}{2}
$$

and so,

$$
\left|\frac{R_{v, n+1}^{d^{n}}(t)}{R_{v, n}^{d^{n}}(t)}-1\right| \leq \frac{1}{2}
$$


or equivalently,

$$
\left(\frac{1}{2}\right)^{1 / d^{n}} \leq \frac{R_{v, n+1}(t)}{R_{v, n}(t)} \leq\left(\frac{3}{2}\right)^{1 / d^{n}}
$$

Taking logarithms in (3.4) and using again a telescoping sum, we obtain the uniform convergence of $\left\{\log R_{v, n}(t)\right\}_{n}$ for all $t \in X\left(\mathbb{C}_{v}\right) \backslash S$ such that $|\psi(t)|_{v}>R$. Finally, using also the convergence at the poles (according to $(3.2)$ ), we conclude the proof of Proposition 3.3 .

3.3. Equidistribution of small points. We use the same construction as in [GHT15, Section 7]. So, from Lemma 3.2 and Proposition 3.3 , we know that

$$
\overline{\mathcal{L}}:=\left(\mathcal{L},\left\{\|\cdot\|_{v}\right\}_{v \in \Omega_{K}}\right),
$$

is an adelic metrized line bundle which is semipositive. The height function $\hat{h}_{\overline{\mathcal{L}}}$ on $X(\bar{K})$ associated to $\overline{\mathcal{L}}$ is given by:

$$
\hat{h}_{\overline{\mathcal{L}}}(t):=\sum_{v \in \Omega_{K}} \frac{N_{v}}{|\operatorname{Gal}(\bar{K} / K) \cdot t|} \cdot \sum_{y \in \operatorname{Gal}(\bar{K} / K) \cdot t}-\log \|s(y)\|_{v}, \text { for any } t \in X(\bar{K})
$$

where $s$ is any section of $\mathcal{L}=\psi^{*} \mathcal{O}_{\mathbb{P}^{1}}(1)$ which does not vanish on the Galois orbits of $t$. The product formula guarantees that this height does not depend on the section $s$ in the above formula.

The adelic metrized line bundle $\overline{\mathcal{L}}$ is uniquely determined by the non-constant morphism $\psi$ : $X \rightarrow \mathbb{P}^{1}$ (defined over $\left.K\right)$. For convenience, we use a new notation for the height $\hat{h}_{\overline{\mathcal{L}}}$ on $X$ associated to the morphism $\psi$ :

$$
\hat{h}_{\psi}(t):=\hat{h}_{\overline{\mathcal{L}}}(t) \text {, for } t \in X(\bar{K}) .
$$

So, as a corollary of Theorem 2.1 applied to the problem we study, we obtain the following equidistribution theorem for points of height tending to 0 .

Theorem 3.4. Let $X$ be a nonsingular projective curve over a number field $K$ and $\psi: X \rightarrow \mathbb{P}^{1}$ be a non-constant morphism defined over $K$. The adelic metrized line bundle $\overline{\mathcal{L}}$ in (3.5), corresponding to the ample line bundle $\mathcal{L}=\psi^{*} \mathcal{O}_{\mathbb{P}^{1}}(1)$ is semipositive. Let $\left\{t_{n}\right\}_{n \geq 1} \subset X(\bar{K})$ be any non-repeating sequence of small points, i.e. $\lim _{n \rightarrow \infty} \hat{h}_{\psi}\left(t_{n}\right)=0$. Then for any place $v \in \Omega_{K}$, the Galois orbits of this sequence are equidistributed in the analytic space $X_{\mathbb{C}_{v}}^{a n}$ with respect to the probability measure $d \mu_{v}=c_{1}(\overline{\mathcal{L}})_{v} / \operatorname{deg}_{\mathcal{L}}(X)$.

Remark 2. We note that $h_{\overline{\mathcal{L}}}(X)=0$ because $X$ contains an infinite set of points with height 0 (see [Zha95b, Theorem (1.10)], Proposition 3.5 and Remark 3).

We obtain next the relation between the two heights $\hat{h}_{\psi}$ and $\hat{h}_{f_{\psi(t)}}$; we recall that $\hat{h}_{f_{\psi(t)}}$ is the canonical height for points on the affine line under the action of the polynomial

$$
f_{\psi(t)}(z):=z^{d}+\psi(t)
$$

Also, we recall that $S$ is the set of poles for $\psi$.

Proposition 3.5. For each $t \in X(\bar{K}) \backslash S$, we have $\hat{h}_{\psi}(t)=\frac{d}{d_{\psi}} \cdot \hat{h}_{f_{\psi(t)}}(0)$, while $\hat{h}_{\psi}(t)=0$ for each $t \in S$. In particular, $\hat{h}_{\psi}(t) \geq 0$ on $X(\bar{K})$ with equality if and only if $t$ is a pole of $\psi$ or $f_{\psi(t)}$ is postcritically finite. 
Proof. First, assume that $t \in X(\bar{K})$ is a pole of $\psi$, i.e. $t \in S$. As $\psi$ is defined over $K$, then the Galois orbit of $t$ is contained in $S$. By the product formula (2.1) together with the definition of metrics at a pole $(3.2)$ and the definition of the height $(3.6)$, we see that $\hat{h}_{\psi}(t)=0$.

Secondly, let $t \in X(\bar{K}) \backslash S$; in this case, the points in the Galois orbit of $t$ are not poles of $\psi$. Let $X_{0}, X_{1}$ be the two canonical sections of $\mathcal{O}_{\mathbb{P}^{1}}(1)$, and pick $u_{0}, u_{1} \in K$ such that the section $u_{0} X_{0}+u_{1} X_{1}$ of $\mathcal{O}_{\mathbb{P}^{1}}(1)$ does not vanish on $[\psi(t): 1] \in \mathbb{P}^{1}(\bar{K})$. For each $y \in \operatorname{Gal}(\bar{K} / K) \cdot t$, this section does not vanish on $\psi(y)$. Define $s:=\psi^{*}\left(u_{0} X_{0}+u_{1} X_{1}\right)$, noting that $s$ does not vanish on the Galois orbits of $t \in X(\bar{K})$. Writing $d_{\psi}:=\operatorname{deg}(\psi)$, we have

$$
\begin{aligned}
\hat{h}_{\psi}(t) & =\sum_{v \in \Omega_{K}} \sum_{y \in \operatorname{Gal}(\bar{K} / K) \cdot t} \frac{-N_{v} \cdot \log \|s(y)\|_{v}}{|\operatorname{Gal}(\bar{K} / K) \cdot t|} \text {, by (3.6) and (3.7) } \\
& =\sum_{v \in \Omega_{K}} \sum_{y \in \operatorname{Gal}(\bar{K} / K) \cdot t} \lim _{n \rightarrow \infty} \frac{N_{v} \cdot \log \max \left\{1,\left|f_{\psi(y)}^{n}(0)\right|_{v}\right\}^{1 /\left(d_{\psi} \cdot d^{n-1}\right)}}{|\operatorname{Gal}(\bar{K} / K) \cdot t|} \text {, by (3.1) and (2.1) } \\
& =\frac{1}{|\operatorname{Gal}(\bar{K} / K) \cdot t|} \lim _{n \rightarrow \infty} \sum_{v \in \Omega_{K}} \sum_{y \in \operatorname{Gal}(\bar{K} / K) \cdot t} \frac{N_{v} \cdot \log ^{+}\left|f_{\psi(y)}^{n}(0)\right|_{v}}{d_{\psi} \cdot d^{n-1}} \\
& =\frac{d}{d_{\psi}} \cdot \hat{h}_{f_{\psi(t)}}(0), \text { by }(2.2) \text { and }(2.3) .
\end{aligned}
$$

The second part of the proposition follows, since $\hat{h}_{f_{\psi(t)}}(0) \geq 0$ with equality if and only if the critical point 0 is preperiodic under iteration of $f_{\psi(t)}$; see CS93.

Remark 3. It is well known that there are infinitely many $t \in \overline{\mathbb{Q}}$ such that $f_{t}(z)=z^{d}+t$ is postcritically finite. Since the morphism $\psi: X \rightarrow \mathbb{P}^{1}$ is non-constant, the set of points with zero height (for the height function $\hat{h}_{\psi}$ ) is Zariski dense on $X(\bar{K})$.

\section{Bifurcation AND POTENTIAL FUnCTiOns}

In this section, we study the bifurcation of algebraic families of unicritical polynomials, parametrized by quasi-projective curves. Let $X^{\prime}$ be (as in the previous Section) a Zariski open dense subset of an irreducible, nonsingular, projective curve $X$ which is a parameter space for two families of unicritical polynomials. In Theorem 4.1 we prove that if there are infinitely many points in $X^{\prime}$ such that the corresponding two polynomials for these two families are simultaneously PCF, then these two families of polynomials have the same normalized bifurcation measure on $X^{\prime}(\mathbb{C})$; this result is a consequence of Theorem 3.4 and the definition of the bifurcation measure (see Subsection 4.3.

4.1. Bifurcation. For a holomorphic family $f(t, \cdot): \mathbb{P}^{1} \rightarrow \mathbb{P}^{1}$ of rational functions of degree $d \geq 2$ parametrized by a complex manifold, we have a stable region, a bifurcation locus (which is the complement of the stable region) and a bifurcation measure (or (1,1)-current) on the parameter space; see [Dem01, Dem03, DF08, Mcm94, MSS83]. One of the main goals in complex dynamics is to study the stability of holomorphic families (or moduli spaces) of rational functions. In this article, we restrict our study to algebraic families of unicritical polynomials, parametrized by quasi-projective curves. 
We work with the notation as in Subsection 3.1. So, $X$ is a smooth, irreducible curve, $X^{\prime}$ is a Zariski dense open subset of $X$, and $f: X^{\prime} \times \mathbb{C} \rightarrow \mathbb{C}$ is an algebraic family of unicritical polynomials of degree $d \geq 2$, i.e. $f_{\psi(t)}(z)=z^{d}+\psi(t)$ where $\psi: X^{\prime} \longrightarrow \mathbb{A}^{1}$ is a morphism. A point $t_{0} \in X^{\prime}$ is stable if the Julia sets $J_{f_{\psi(t)}}$ are moving holomorphically in a neighbourhood of $t_{0}$, or equivalently, $\left\{f_{\psi(t)}^{n}(0)\right\}_{n \geq 1}$ is a normal family of functions on some neighbourhood of $t_{0}$. The bifurcation locus on $X^{\prime}(\mathbb{C})$ is the set of parameters where $f_{\psi(t)}$ fails to be stable. By definition, the stable region is always an open subset of $X^{\prime}(\mathbb{C})$.

We define the escape-rate function for $\psi$ as

$$
G_{\psi}(t):=\lim _{n \rightarrow \infty} \frac{1}{d^{n}} \log ^{+}\left|f_{\psi(t)}^{n}(0)\right|,
$$

which is a subharmonic function on $X^{\prime}(\mathbb{C})$. It is convenient to extend the function $G_{\psi}$ on $X(\mathbb{C})$ by defining it

$$
G_{\psi}(t)=0 \text { for each } t \in\left(X \backslash X^{\prime}\right)(\mathbb{C}) .
$$

The differential of the bifurcation measure is defined as

$$
d \mu_{\psi}:=d d^{c} G_{\psi}(t)
$$

with $d d^{c}=\frac{i}{\pi} \partial \bar{\partial}$ being the Laplacian operator. For the sake of simplifying the notation, when $\psi(t)=t$ is the identity map, we use $\mu$ and $G(t)$ instead of $\mu_{\psi}$ and $G_{\psi}(t)$. The support of the bifurcation measure coincides with the bifurcation locus on $X^{\prime}(\mathbb{C})$, and the bifurcation locus is empty if and only if $\psi$ is a constant (i.e. $f_{\psi(t)}$ is isotrivial). From the definition of the the escape-rate function, we see that

$$
G_{\psi}(t)=G(\psi(t))
$$

i.e. $G_{\psi}=\psi^{*} G$ is the pullback of the escape-rate function on the complex plane by $\psi$. Hence the bifurcation measure (resp. bifurcation locus) is the pullback of the bifurcation measure (resp. bifurcation locus) on the complex plane

$$
\mu_{\psi}=\psi^{*} \mu
$$

i.e. $\mu_{\psi}(A)=\mu(\psi(A))$ for $A \subset X^{\prime}(\mathbb{C})$ with $\psi$ being injective on $A$.

4.2. The generalized Mandelbrot sets. Here we deal with the simplest case: $\psi(t)=t$ (i.e. $\left.f_{\psi(t)}(z)=f_{t}(z)=z^{d}+t\right)$ and $X^{\prime}$ itself is the affine (complex) line. The degree $d$ generalized Mandelbrot set $\mathcal{M}_{d}$ is the set of parameters where the critical point 0 is bounded under the iterates of $f_{t}$

$$
\mathcal{M}_{d}:=\left\{t \in \mathbb{C}:\left|f_{t}^{n}(0)\right| \not \rightarrow \infty \text { as } n \rightarrow \infty\right\}
$$

When $d=2, \mathcal{M}_{2}$ is the classical Mandelbrot set. See Figure 5.1 for the pictures of $\mathcal{M}_{2}$ and $\mathcal{M}_{3}$. We recall some basic properties of the generalized Mandelbrot sets. Every generalized Mandelbrot set is bounded and simply connected, and there is a unique biholomorphic map $\Phi$ (depending on d) from $\mathbb{C} \backslash \mathcal{M}_{d}$ to the complement of the closed unit disk $\mathbb{C} \backslash \overline{\mathbb{D}}$

$$
\Phi: \mathbb{C} \backslash \mathcal{M}_{d} \stackrel{\sim}{\longrightarrow} \mathbb{C} \backslash \overline{\mathbb{D}}
$$

with $\Phi(t)=t+O(1)$, for $|t|>>0$. The Green's function $G_{\mathcal{M}_{d}}$ for the compact set $\mathcal{M}_{d}$ on $\mathbb{C} \backslash \mathcal{M}_{d}$ is given by

$$
G_{\mathcal{M}_{d}}(t)=\log |\Phi(t)|
$$


and it is known that $G_{\mathcal{M}_{d}}(t)=d \cdot G(t)$ (for example, see [BD11]). Moreover, the escape-rate function satisfies the inequality $G(t) \geq 0$ with equality if and only if $t \in \mathcal{M}_{d}$. The bifurcation locus for $f_{t}$ is the boundary $\partial \mathcal{M}_{d}$ of $\mathcal{M}_{d}$, and the bifurcation measure is proportional to the harmonic measure for $\mathcal{M}_{d}$.

4.3. Two algebraic families of unicritical polynomials. Let $X$ be a nonsingular projective curve defined over a number field $K$, let $\psi: X \longrightarrow \mathbb{P}^{1}$ be a non-constant morphism, and let $S \subset X$ be its set of poles. We proceed as in subsection 3.2 and define the adelic metrized line bundle $\overline{\mathcal{L}}$ endowed with metrics $\|\cdot\|_{v}$ for each $v \in \Omega_{K}$. We recall that when $v$ is archimedean, $\mathbb{C}_{v} \cong \mathbb{C}$ and $X_{\mathbb{C}_{v}}^{a n} \cong X(\mathbb{C})$. The curvature $c_{1}(\overline{\mathcal{L}})_{v}$ of $\|\cdot\|_{v}$ is given by $c_{1}(\overline{\mathcal{L}})_{v}=-d d^{c} \log \|\cdot\|_{v}$.

For the rest of this subsection, we fix an archimedean place $v$ and identify $\mathbb{C}_{v}$ with $\mathbb{C}$. For $t_{0} \in X(\mathbb{C}) \backslash S$, we let $s$ be a section on $\mathbb{P}^{1}$ defined over $K$ which does not vanish at $\psi\left(t_{0}\right)$. Hence for $t \in X(\mathbb{C})$ in a neighbourhood of $t_{0}$, using $(3.1)$, we have

$$
c_{1}(\overline{\mathcal{L}})_{v}(t)=-d d^{c} \log \left\|\psi^{*}(s)\left(t_{0}\right)\right\|_{v}=d d^{c} \lim _{n \rightarrow \infty} \frac{\log ^{+}\left|f_{\psi(t)}^{n}(0)\right|_{v}}{d_{\psi} \cdot d^{n-1}}=\frac{d}{d_{\psi}} \cdot d d^{c} G_{\psi}(t) .
$$

For the bifurcation measure $\mu_{\psi}$ on $X(\mathbb{C}) \backslash S$, we have

$$
\mu_{\psi}=\frac{d_{\psi}}{d} \cdot \mu_{v}
$$

where $d_{\psi}$ is the degree of $\psi$. In particular, we consider $\mu_{v}$ be the normalized bifurcation measure with respect to which we get the equidistribution statement from Theorem 3.1 .

Proof of Theorem 3.1. Let $\left\{t_{n}\right\} \subset X^{\prime}(\mathbb{C})$ be a sequence of PCF parameters for the algebraic family $f: X^{\prime} \times \mathbb{C} \longrightarrow \mathbb{C}$ of unicritical polynomials of degree $d$. First of all, we note that each $\psi\left(t_{n}\right) \in \overline{\mathbb{Q}}$ since $z^{d}+\psi\left(t_{n}\right)$ is a PCF map; since $\psi$ is defined over $\overline{\mathbb{Q}}$, then also $t_{n} \in \overline{\mathbb{Q}}$. Then by Proposition 3.5 .

$$
\hat{h}_{\psi}\left(t_{n}\right)=0=\hat{h}_{f_{\psi\left(t_{n}\right)}}(0) .
$$

Using Theorem 3.4, we conclude that the points $\left\{t_{n}\right\}$ equidistribute with respect to $\mu_{v}$, as desired.

Now, we consider two non-constant morphisms $\psi_{i}: X \rightarrow \mathbb{P}^{1}$ for $i=1,2$, with sets of poles $S_{1}$ and $S_{2}$, respectively. They determine two algebraic families of unicritical polynomials $f_{\psi_{1}(t)}$ and $f_{\psi_{2}(t)}$ of degree $d \geq 2$.

Theorem 4.1. Suppose there are infinitely many $t \in X \backslash\left(S_{1} \cup S_{2}\right)$, such that $f_{\psi_{1}(t)}$ and $f_{\psi_{2}(t)}$ are simultaneously postcritically finite. Then $d_{\psi_{2}} \cdot \mu_{\psi_{1}}=d_{\psi_{1}} \cdot \mu_{\psi_{2}}$ on $X \backslash\left(S_{1} \cup S_{2}\right)$. Furthermore, on $X(\mathbb{C})$

$$
d_{\psi_{2}} \cdot G_{\psi_{1}}(t)=d_{\psi_{1}} \cdot G_{\psi_{2}}(t)
$$

Proof. The relations of the two bifurcation measures is clear from Theorem 3.4 and (4.4). And then these two families have the same stable region on $X(\mathbb{C}) \backslash\left(S_{1} \cup S_{2}\right)$. Let

$$
H(t):=d_{\psi_{2}} \cdot G_{\psi_{1}}(t)-d_{\psi_{1}} \cdot G_{\psi_{2}}(t)
$$

be the difference of the two continuous subharmonic functions on $X(\mathbb{C}) \backslash\left(S_{1} \cup S_{2}\right)$. Since $d_{\psi_{2}} \cdot \mu_{\psi_{1}}=$ $d_{\psi_{1}} \cdot \mu_{\psi_{2}}$, and also using (4.1), $H(t)$ is harmonic on $X(\mathbb{C}) \backslash\left(S_{1} \cup S_{2}\right.$ ). The pullback (by $\psi_{1}$ or $\psi_{2}$ ) of a connected component of the stable region $\mathbb{C} \backslash \partial \mathcal{M}_{d}$, consists of finitely many (up to the 
degree $d_{\psi_{1}}$ or $d_{\psi_{2}}$ ) connected components of the stable region on $X$. As $\mathbb{C} \backslash \partial \mathcal{M}_{d}$ consists of infinitely many connected components (see Figure 5.1), so is $\psi_{2}^{-1}\left(\mathbb{C} \backslash \partial \mathcal{M}_{d}\right)$. Then we can pick one connected component of the stable region on $X(\mathbb{C}) \backslash\left(S_{1} \cup S_{2}\right)$, such that its images under both $\psi_{1}$ and $\psi_{2}$ are stable subsets within the generalized Mandelbrot set $\mathcal{M}_{d}$. Hence for any $t$ in this component, $G_{\psi_{1}}(t)=G\left(\psi_{1}(t)\right)=0=G\left(\psi_{2}(t)\right)=G_{\psi_{2}}(t)$, which yields that $H(t)=0$. So the harmonic function $H(t)$ on $X(\mathbb{C}) \backslash\left(S_{1} \cup S_{2}\right)$, which is identically zero on some open subset of $X(\mathbb{C}) \backslash\left(S_{1} \cup S_{2}\right)$, must be zero everywhere, and so 4.5 follows.

Remark 4. The set $S$ of poles for $\psi$ is the set of points $t_{0} \in X$ such that $\lim _{t \rightarrow t_{0}} G_{\psi}(t)=\infty$. With the same assumptions as in Theorem 4.1, one has $S_{1}=S_{2}$ for the sets of poles of $\psi_{1}$ and $\psi_{2}$. And moreover, by (4.3) (4.5), for any $t \in X(\mathbb{C})$ with $\psi_{1}(t) \in \mathbb{C} \backslash \mathcal{M}_{d}$ (hence $\psi_{2}(t) \in \mathbb{C} \backslash \mathcal{M}_{d}$ by proportionality of $G_{\psi_{1}}$ and $G_{\psi_{2}}$ ), we have

$$
\left|\Phi\left(\psi_{1}(t)\right)\right|^{d_{\psi_{2}}}=\left|\Phi\left(\psi_{2}(t)\right)\right|^{d_{\psi_{1}}} .
$$

\section{Proof of the MAIN THEOREM}

Suppose now that $X$ is an irreducible, nonsingular projective curve satisfying the hypothesis of Theorem 4.1. By Remark 4, we conclude that for all $t \in X(\mathbb{C})$,

$$
\psi_{1}(t) \in \mathbb{C} \backslash \mathcal{M}_{d} \Leftrightarrow \psi_{2}(t) \in \mathbb{C} \backslash \mathcal{M}_{d},
$$

and further that the uniformizing map $\Phi: \mathbb{C} \backslash \mathcal{M}_{d} \rightarrow \mathbb{C} \backslash \overline{\mathbb{D}}$ satisfies

$$
\left|\Phi\left(\psi_{1}(t)\right)\right|^{d_{\psi_{2}}}=\left|\Phi\left(\psi_{2}(t)\right)\right|^{d_{\psi_{1}}} .
$$

Write $d_{1}=d_{\psi_{1}}, d_{2}=d_{\psi_{2}}$. Let $X_{0}$ be a connected, unbounded component of the stable region in $X$; i.e., $X_{0}$ is a component of the preimage of $\mathbb{C} \backslash \mathcal{M}_{d}$ under $\psi_{2}$. The quotient

$$
\Phi\left(\psi_{1}(t)\right)^{d_{2}} / \Phi\left(\psi_{2}(t)\right)^{d_{1}}
$$

provides a holomorphic map $X_{0} \rightarrow S^{1}$ (where $S^{1}$ is the complex unit circle); by the Open Mapping Theorem, this map is constant, so there exists $\eta \in \mathbb{R}$ such that for all $t \in X_{0}$,

$$
\Phi\left(\psi_{1}(t)\right)^{d_{2}}=e^{2 \pi i \eta} \cdot \Phi\left(\psi_{2}(t)\right)^{d_{1}}
$$

Following $[\mathrm{DH}]$, the standard tool for studying the behavior of the degree $d$ Mandelbrot set $\mathcal{M}_{d}$ is given by the external rays of the map $\Phi$. We define the external ray for an angle $\theta \in \mathbb{R} / \mathbb{Z}$ to be

$$
\mathcal{R}(\theta):=\Phi^{-1}\left(\left\{r e^{2 \pi i \theta}: r>1\right\}\right) .
$$

We recall some standard facts about external rays; see Chapters 8 and 13 of [DH], and [EMS]. An external ray $\mathcal{R}(\theta)$ is said to be rational if $\theta$ is rational. A point $c \in \mathcal{M}$ is Misiurewicz if the critical point 0 of $z^{d}+c$ is strictly preperiodic, and clearly every PCF point on the boundary of $\mathcal{M}_{d}$ is a Misiurewicz point.

Proposition 5.1. [DH] All rational rays land; that is, there exists a unique point $c_{\theta} \in \partial \mathcal{M}_{d}$ such that $\lim _{r \rightarrow 1} \Phi^{-1}\left(r e^{2 \pi i \theta}\right)=c_{\theta}$. Misiurewicz points are contained in the boundary of $\mathcal{M}_{d}$, and every Misiurewicz point is the landing point of at least one rational ray. 

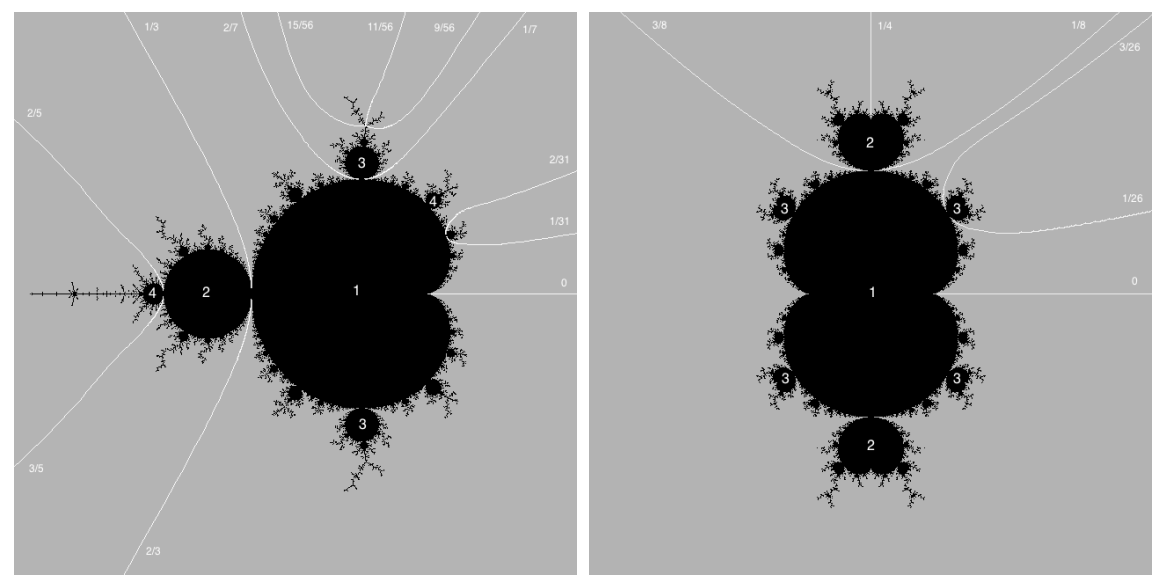

FiguRE 5.1. Selected external rays of the degree 2 (left) and degree 3 (right) Mandelbrot sets. Angles of each ray are indicated next to the ray. A select number of hyperbolic components are labeled with the period of the component. External rays drawn by Wolf Jung's program Mandel.

Let $\alpha$ be a periodic point of $f_{c}(z)=z^{d}+c$ with exact period $n$. The multiplier of this cycle is $\lambda:=\left(f_{c}^{n}\right)^{\prime}(\alpha)$. The cycle is attracting if $|\lambda|<1$, repelling if $|\lambda|>1$, and parabolic if $\lambda$ is a root of unity. A parameter $c$ is parabolic if $f_{c}(z)=z^{d}+c$ contains a parabolic cycle; in this case, there is a unique parabolic cycle. Parabolic points also lie in $\partial \mathcal{M}_{d}$, and are the landing points of rational rays.

Proposition 5.2. $[\mathrm{DH}]$, EMS Every parabolic point $c$ is the landing point of either one or two rational rays. If the parabolic cycle of $f_{c}(z)$ has multiplier $\lambda \neq 1$, then exactly two distinct rational rays land at $c$.

If $\mathcal{R}(\theta)$ and $\mathcal{R}\left(\theta^{\prime}\right)$ land at the same point, we say $\theta$ and $\theta^{\prime}$ are a landing pair. If their common landing point $c$ is parabolic with multiplier $\neq 1$, then $\mathcal{M}_{d} \backslash\{c\}$ consists of two connected components. In this case, the component which does not contain 0 is the wake $w_{c}$ of $c$, and if $\mathcal{R}(\theta)$ and $\mathcal{R}\left(\theta^{\prime}\right)$ land at $c$, the width of the wake $w_{c}$ is defined to be $\left|w_{c}\right|:=\theta^{\prime}-\theta$, assuming $0<\theta<\theta^{\prime}<1$. For more about external rays, one can refer to [EMS]. For illustration, see Figure 5.1 .

Recall that a stable, connected component $H$ in $\mathcal{M}_{d}$ is hyperbolic of period $\ell$ if $z^{d}+c$ has an attracting cycle of exact period $\ell$ for every $c \in H$.

Proposition 5.3. For all $k \geq 1, \frac{1}{d^{k}-1}$ and $\frac{d}{d^{k}-1}$ are a landing pair, and their landing point $c_{k}$ lies on the boundary of both the unique period 1 hyperbolic component, and a component of period $k$.

The proof of the proposition is by standard arguments; see Proposition 3.5 of [GKN] for details.

A hyperbolic component $H$ in $\mathcal{M}_{d}$ is equipped with a $(d-1)$-to- 1 map $\lambda_{H}: H \rightarrow \mathbb{D}$, given by the multiplier of the attracting cycle; this map extends continuously to the boundary; the point 0 has a unique preimage under the multiplier map, known as the center of the hyperbolic component. Given $\frac{p}{q} \in \mathbb{Q} / \mathbb{Z}$, the preimage under $\lambda_{H}$ of the ray $\left\{r e^{2 \pi i p / q}: 0<r<1\right\} \subset \mathbb{D} \backslash\{0\}$ is a collection of $d-1$ disjoint curves in the component (known as internal rays), which land at 


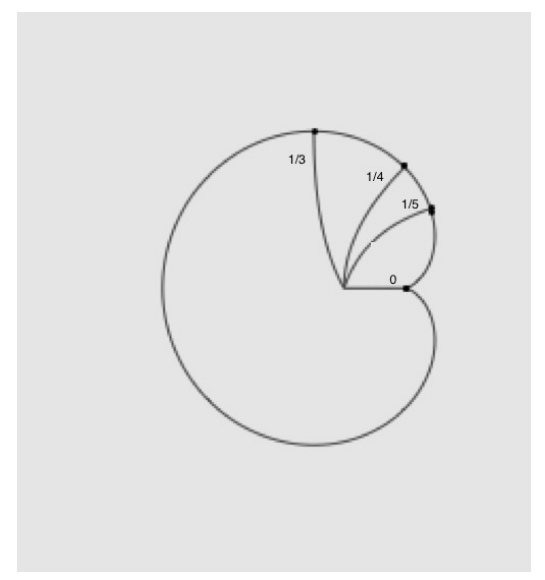

FigURE 5.2. Select internal rays of the period 1 component of the Mandelbrot set, with angles indicated. Internal rays drawn by Wolfram's Mathematica.

parabolic points on $\partial H$. In this case, we say that the wake is a $\frac{p}{q}$-subwake of $H$. Conversely, each parabolic point is the landing of some internal ray. See Figure 5.2 for an illustration.

For example, if $H$ is the period 1 component, some preimage of the point $e^{2 \pi i / k} \in \partial \mathbb{D}$ will land at the point $c_{k} \in \partial H$ of Proposition 5.3 .

If $H$ is a hyperbolic component of $\mathcal{M}_{d}$ of period $>1$, there is a unique point $c_{H}$ on the boundary of $H$ so that both $\lambda\left(c_{H}\right)=1$ and $\mathcal{M}_{d} \backslash\left\{c_{H}\right\}$ consists of two connected components; this is the root of $H$. There will be exactly two rays landing at $c_{H}$, and we correspondingly define the width of the component $H$ to be the width of the wake at $c_{H}$.

Our key tool towards the main theorem is the so-called wake formula, which was folklore, eventually due to Bruin-Kaff-Schleicher in [BKL09] for $d=2$ and Kauko for general $d$ (see [Kau00]):

Proposition 5.4. Let $H$ be a hyperbolic component of $\mathcal{M}_{d}$ with period $k$ and width $|H|$. Let $w_{p / q}$ be any $\frac{p}{q}$-subwake of $H$. Then

$$
\left|w_{p / q}\right|=\frac{|H|}{d-1} \frac{\left(d^{k}-1\right)^{2}}{d^{q k}-1} .
$$

Corollary 5.5. Let $H$ be a hyperbolic component of the degree $d$ Mandelbrot set. Then the 1/2-subwakes of $H$ are precisely the set of subwakes of $H$ with maximal possible width.

We provide now a key proposition towards Theorem 1.1 .

Proposition 5.6. Under the hypothesis of Theorem 4.1, there exists a component $X_{0}$ of the preimage of $\mathbb{C} \backslash \mathcal{M}_{d}$ under $\psi_{2}$ such that the real number $\eta$ of Equation 5.1 is rational.

Proof. We have two cases. Suppose first that there exists $t \in X$ satisfying the following:

(1) $t_{0}$ is not a branch point for $\psi_{1}$ or $\psi_{2}$,

(2) both $\psi_{1}(t)$ and $\psi_{2}(t)$ are PCF parameters, and

(3) $\psi_{1}(t)$ or $\psi_{2}(t)$ is a Misiurewicz point.

Suppose without loss of generality that $\psi_{1}(t)$ is Misiurewicz; since $\psi_{1}(t) \in \partial \mathcal{M}_{d}$, every open neighborhood of $\psi_{1}(t)$ contains parameters $c$ such that $|\Phi(c)|>0$; by Equation 5.1, the same 
holds for $\psi_{2}(t)$. Choose $X_{0}$ so that $t \in X_{0}$, and write $\mathcal{R}\left(\theta_{1}\right)$ and $\mathcal{R}\left(\theta_{2}\right)$ for the rational external rays landing at $\psi_{1}(t)$ and $\psi_{2}(t)$, respectively. By Equation 5.1, we have

$$
d_{2} \theta_{1}-d_{1} \theta_{2}=\eta
$$

and so $\eta$ is rational as desired.

Suppose now that the conditions above are not satisfied for any $t \in X$. Call $B$ the set of branch points of the projection maps $\psi_{i}$. Since $B$ is a finite set, then by hypothesis, there exists some $t_{0} \in X \backslash B$ such that both $\psi_{1}\left(t_{0}\right)$ and $\psi_{2}\left(t_{0}\right)$ are centers of hyperbolic components. In fact, we may choose $t_{0}$ so that the component $H_{2}$ of $\mathcal{M}_{d}$ which has center $\psi_{2}\left(t_{0}\right)$ is far from the branch points in the following sense: there exists an open neighborhood $U$ of $t_{0}$ such that $\psi_{2}(U)$ is simply connected, $\mathrm{H}_{2} \subset \psi_{2}(U)$, and there exists a parabolic parameter $c \in \partial H_{2}$ such that $c$ is the root of a component $H_{2}^{\prime}$ satisfying $\overline{H_{2}^{\prime}} \subset \psi_{2}(U)$. Therefore we have a well-defined analytic function

$$
h(z):=\psi_{1} \circ \psi_{2}^{-1}: U \rightarrow h(U) .
$$

Since $h$ is an open map, $h\left(H_{2}\right)$ is a component of $\mathbb{C} \backslash \partial \mathcal{M}_{d}$ which contains the PCF parameter $\psi_{1}\left(t_{0}\right)$, so is hyperbolic. Since $U$ contains no branch points, $h(c)$ lies on the boundary of two stable components, so by Theorem 4.1 of [Sch04, $h(c)$ is a parabolic parameter. Choose $t \in X$ such that $\psi_{1}(t)=h(c)$ and $\psi_{2}(t)=c$, and choose $X_{0}$ so that $t \in X_{0}$. By Equation 5.1, any rational rays $\mathcal{R}(\theta)$ landing at $c$ and $\mathcal{R}\left(\theta^{\prime}\right)$ landing at $h(c)$ satisfy the relation

$$
d_{2} \theta^{\prime}-d_{1} \theta=\eta
$$

and we conclude that $\eta$ is rational as desired.

We are now ready to prove the remaining significant result towards Theorem 1.1 .

Theorem 5.7. Assume the hypothesis of Theorem 4.1. Then there exists an open subset $U$ of the complex plane containing infinitely many PCF parameters on which an analytic branch of $\psi_{1} \circ \psi_{2}^{-1}$ is given by $z \mapsto \zeta z$, for some $(d-1)$ st root of unity $\zeta$.

Proof. Fix an integer $m>2$. We will define a neighborhood $U(m)$ as follows: define $c_{m}$ to be the landing point of the external ray $\mathcal{R}\left(\frac{d}{d^{m}-1}\right)$; by Proposition 5.3 , this point lies on the main component. By the preceding discussion, there also exists an internal ray $r_{m}$ landing at $c_{m}$, as well the internal ray $r_{0}$ of angle zero which lands at $c_{0}=(d-1) / d^{d /(d-1)}$. The union

$$
C:=r_{0} \cup r_{m} \cup \mathcal{R}(0) \cup \mathcal{R}\left(\frac{d}{d^{m}-1}\right) \cup\{0\}
$$

is a curve such that $\mathbb{C} \backslash C$ has two (simply connected) components. Define $U(m)$ to be the component of $\mathbb{C} \backslash C$ which contains the hyperbolic component with root $c_{m}$; in other words, the component containing parameters of arbitrarily small argument (see Figure 5.1, 5.2). By Proposition 5.3, $U(m)$ contains infinitely many PCF parameters.

Note now that $m$ may be chosen sufficiently large so that $U(m)$ omits the images of the branch points of $\psi_{1}$ and $\psi_{2}$. Therefore we may define an analytic branch $h(z)=\psi_{1} \circ \psi_{2}^{-1}$ on $U(m)$. By Equation 5.1, $h$ sends external rays to external rays, and there exists $0<\ell \leq d_{2}$ (given by choice 
of branch) such that $h$ acts on external angles $0<\theta<\frac{d}{d^{m}-1}$ by

$$
\theta \mapsto \frac{d_{1}}{d_{2}} \theta+\eta+\frac{\ell}{d_{2}}
$$

Denote such a choice of $U(m)$ simply by $U$, and write $\eta+\frac{\ell}{d_{2}}=\frac{a}{b}$ in lowest terms (this is possible by Proposition 5.6). Note then that if $\mathcal{R}\left(\theta_{1}\right)$ and $\mathcal{R}\left(\theta_{2}\right)$ land together in $U$, their images under the continuous map $h$ must land together, and the wake of the image rays has width $\frac{d_{1}}{d_{2}}\left|\theta_{2}-\theta_{1}\right|$.

Proposition 5.8. Let $U$ and $h$ be defined as above. Then every hyperbolic component of $U$ is sent by $h$ to a hyperbolic component of the same period.

Proof of Proposition 5.8. Suppose $H$ is a hyperbolic component of period $N$ contained in the neighborhood $U$ with root $c$ and wake $W$. Since $h$ is an open map on $U$ which preserves rational external rays, $h(H)$ is also a hyperbolic component, say of period $N^{\prime}$, root $h(c)$, and wake $h(W)$. Choose any landing point of an internal ray of $H$ with angle $1 / 2$; call this point $c_{1 / 2}$, and its subwake $H_{1 / 2}$, noting that $c_{1 / 2}$ is the root of a hyperbolic component of period $2 N$. Since $h$ has a linear action on external angles, Corollary 5.5 guarantees that $h\left(H_{1 / 2}\right)$ is one of the $1 / 2$-subwakes of $h(H)$; call it $h(H)_{1 / 2}$. The width formula of Proposition 5.4 computes:

$$
\frac{|h(H)|}{d-1} \frac{\left(d^{N^{\prime}}-1\right)^{2}}{d^{2 N^{\prime}}-1}=\left|h(H)_{1 / 2}\right|=\left|h\left(H_{1 / 2}\right)\right|=\frac{d_{1}}{d_{2}}\left|H_{1 / 2}\right|=\frac{d_{1}}{d_{2}} \frac{|H|}{d-1} \frac{\left(d^{N}-1\right)^{2}}{d^{2 N}-1} .
$$

Since $|h(H)|=\frac{d_{1} \ell}{d_{2}}|H|$, the right- and left-hand sides of the equation above imply that $N=N^{\prime}$, as desired.

For any hyperbolic component of period $N$, the rays landing at the root of the component have denominators which divide $d^{N}-1$ (this is again standard; see Chapter 8 of [DH]), and therefore the width of a period $N$ component has denominator which is a divisor of $d^{N}-1$. Since $h$ fixes the period of any hyperbolic component in $U$, and $U$ contains components of period $N$ and width $\frac{d-1}{d^{N}-1}$ for all $N>m$ (see Proposition 5.3), we deduce that $\frac{d_{1}}{d_{2}} \cdot \frac{d-1}{d^{N}-1}$ has denominator which is a divisor of $d^{N}-1$ for all $N>m$. We conclude that $d_{2}$ divides $d_{1}(d-1)$, and so the map that $h$ induces on $\mathbb{R} / \mathbb{Z} \cap\left(0, \frac{d}{d^{m}-1}\right)$ is simply

$$
\theta \mapsto \frac{k}{d-1} \theta+\frac{a}{b}
$$

for some $1 \leq k \leq d-1$.

By the same arguments and choosing $N$ sufficiently large, the ray

$$
h\left(\mathcal{R}\left(\frac{1}{d^{N}-1}\right)\right)=\mathcal{R}\left(\frac{d-1}{d^{N}-1} \cdot \frac{k}{d-1}+\frac{a}{b}\right)
$$

lands at a hyperbolic component of period $N$, and so $\frac{k}{d^{N}-1}+\frac{a}{b}$ has denominator dividing $d^{N}-1$. Since $a / b$ is in lowest terms, we conclude that $b$ divides $d^{N}-1$ for all $N$ sufficiently large. Choosing $M$ and $N$ large and coprime, the greatest common divisor of $d^{M}-1$ and $d^{N}-1$ is $d-1$, so we have $b \mid(d-1)$.

We now have integers $k, j$ so that $h$ acts on external angles of $U$ by

$$
\theta \mapsto \frac{k}{d-1} \theta+\frac{j}{d-1}
$$


However, we know that the ray of angle $\frac{1}{d^{N}-1}$ maps to a ray with denominator dividing $d^{N}-1$ for all $N$ sufficiently large; in other words,

$$
k+j\left(d^{N}-1\right) \equiv 0 \bmod (d-1)
$$

for all $N$ sufficiently large. Thus $k=d-1$, and so $h$ acts on external angles by translation by $\frac{j}{d-1}$; that is, $h$ acts on external rays as multiplication by some $(d-1)$ st root of unity $\zeta$.

By definition of $\Phi$,

$$
\Phi(\zeta z)=\zeta \Phi(z)
$$

for all $z \in \mathbb{C} \backslash \mathcal{M}_{d}$, so $h$ coincides with the map $z \mapsto \zeta z$ on $\left(\mathbb{C} \backslash \mathcal{M}_{d}\right) \cap U$, and thus on the entire domain $U$.

The proof of the main theorem follows easily from Theorem 5.7 .

Proof of Theorem 1.1. First we note that indeed, if $C$ has the form (1), (2) or (3) as in the conclusion of Theorem 1.1, then it contains infinitely many points $(a, b)$ with both coordinates PCF parameters, i.e., both $z^{d}+a$ and $z^{d}+b$ are PCF polynomials. For curves of the form (1) or (2), this fact is obvious, while for curves of the form (3), we note that once $f_{c}(z):=z^{d}+c$ is PCF, then also $f_{\zeta c}(z):=z^{d}+\zeta c$ is PCF (where $\zeta^{d-1}=1$ ) because $\zeta^{-1} f_{\zeta c}(\zeta z)=f_{c}(z)$. Also, there exist infinitely many $c \in \overline{\mathbb{Q}}$ such that $f_{c}$ is PCF.

So, from now on, assume $C$ be an irreducible plane curve containing infinitely many $(a, b)$ such that $z^{d}+a$ and $z^{d}+b$ are both PCF. Since the PCF parameters are algebraic numbers, we conclude that $C$ is defined over $\overline{\mathbb{Q}}$. If $C$ does not project dominantly onto one of the two coordinates of $\mathbb{A}^{2}$ then, without loss of generality, we may assume $C=\left\{t_{0}\right\} \times \mathbb{A}^{1}$ for some $t_{0} \in \overline{\mathbb{Q}}$. But then by the hypothesis satisfied by $C$, we conclude that $t_{0}$ is a PCF parameter, i.e., $C$ has the form (1) as in the conclusion of Theorem 1.1 .

So, from now on, we assume $C$ projects dominantly onto both coordinates of $\mathbb{A}^{2}$. Let $\pi: X \rightarrow C$ be a nonsingular projective model of $C$; therefore $X$ is defined over some number field $K$. Write $\pi_{1}$ and $\pi_{2}$ for the projection maps of $C$ onto the axis of $\mathbb{A}^{2}$, and let $\psi_{i}=\pi_{i} \circ \pi$ for $i=1$, 2 . Then we can apply Theorem 4.1 and deduce Theorem 5.7. Thus there exists a $(d-1)$ st root of unity $\zeta$ such that for infinitely many $c \in \mathcal{M}_{d}, \zeta c=\psi_{1}\left(t_{c}\right)$ and $c=\psi_{2}\left(t_{c}\right)$ for some $t_{c} \in X(\mathbb{C})$; accordingly, there exist infinitely many $c \in \mathbb{C}$ such that $(\zeta c, c) \in C(\mathbb{C})$. Since $C$ is irreducible, we conclude the proof of Theorem 1.1 .

\section{REFERENCES}

[And98] Y. André, Finitude des couples d'invariants modulaires singuliers sur une courbe algébrique plane non modulaire, J. Reine Angew. Math. 505 (1998), 203-208.

[BD11] M. Baker and L. DeMarco, Preperiodic points and unlikely intersections, Duke Math. J. 159 (2011), 1-29.

[BD13] M. Baker and L. DeMarco, Special curves and postcritically-finite polynomials, Forum Math. PI 1 (2013), e3, 35 pages.

[BR06] M. Baker and R. Rumely, Equidistribution of small points, rational dynamics, and potential theory, Ann. Inst. Fourier (Grenoble) 56(3) (2006), 625-688.

[BMZ99] E. Bombieri, D. Masser and U. Zannier, Intersecting a curve with algebraic subgroups of multiplicative groups, Int. Math. Res. Not. 1999 (1999), 1119-1140.

[BKL09] H. Bruin, A. Kaffl and D. Schleicher, Existence of quadratic Hubbard trees, Fund. Math. 202 (2009), 251-279. 
[CL06] A. Chambert-Loir, Mesures etéquidistribution sur les espaces de Berkovich, J. Reine Angew. Math. 595 (2006), 215-235.

[CS93] G. Call and J. Silverman, Canonical heights on varieties with morphisms, Compositio Math. 89(1993), 163-205.

[Dem01] L. DeMarco,Dynamics of rational maps: a current on the bifurcation locus, Math. Res. Lett. 8 (2001), $57-66$.

[Dem03] L. DeMarco, Dynamics of rational maps: Lyapunov exponents, bifurcations, and capacity, Math. Ann. 326 (2003), 43-73.

[Dem] L. DeMarco, Bifurcations, intersections, and heights, Preprint, 2014.

$[\mathrm{DH}] \quad$ A. Douady and J.H. Hubbard, Étude dynamique des polynomes complexes, Publications Mathématiques d'Orsay, 1984-1985.

[DF08] R. Dujardin and C. Favre, Distribution of rational maps with a preperiodic critical point, Amer. J. Math. 130 (2008), 979-1032.

[EMS] D. Eberlein, S. Mukherjee and S. Schleicher, Rational parameter rays of the Multibrot sets, Preprint, 2014.

[FRL06] C. Favre and J. Rivera-Letelier, Équidistribution quantitative des points de petite hauteur sur la droite projective, Math. Ann. 355 (2006), 311-361.

[GHT12] D. Ghioca, L.-C. Hsia and T. J. Tucker, Preperiodic points for families of polynomials, Algebra \& Number Theory 7 (2012), 701-732.

[GHT15] D. Ghioca, L. Hsia and T. Tucker, Preperiodic points for families of rational maps, Proc. London Math. Soc. 110 (2015), 395-427.

[GKN] D. Ghioca, H. Krieger and K. Nguyen, A case of the Dynamical André-Oort Conjecture, to appear in the Intern. Math. Res. Not., 2014.

[Jon13] R. Jones, Galois representations from pre-image trees: an arboreal survey, Pub. Math. Besançon, 2013, 107-136.

[Kau00] V. Kauko, Trees of visible components in the Mandelbrot set, Fund. Math. 164 (2000), 41-60.

[MZ10] D. Masser and U. Zannier, Torsion anomalous points and families of elliptic curves, Amer. J. Math. 132 (2010), 1677-1691.

[MZ12] D. Masser and U. Zannier, Torsion points on families of squares of elliptic curves, Math. Ann. 352 (2012), 453-484.

[Mcm94] C. McMullen, Complex Dynamics and Renormalization, Princeton University Press, Princeton, NJ, 1994.

[MSS83] R. Mañé, P. Sad and D. Sullivan, On the dynamics of rational maps, Ann. Sci. Ec. Norm. Sup. 16 (1983), 193-217.

[Pina] R. Pink, Profinite iterated monodromy groups arising from quadratic morphisms with infinite postcritical orbits, preprint, 2013, available on arxiv.

[Pinb] R. Pink, Profinite iterated monodromy groups arising from quadratic polynmials, preprint, 2013, available on arxiv.

[Pinc] R. Pink, Finiteness and liftability of postcritically finite quadratic morphisms in arbitrary characteristic, preprint, 2013, available on arxiv.

[Sch04] D. Schleicher, On fibers and local connectivity of Mandelbrot and Multibrot sets, Fractal Geometry and Applications: A Jubilee of Benoît Mandelbrot, Proc. Sympos. Pure Math. 72, Part 1, Amer. Math. Soc., Providence, RI, 2004, pp. 477-507.

[Yua08] X. Yuan, Big line bundles over arithmetic varieties, Invent. Math. 173 (2008), 603-649.

[Zan12] U. Zannier, Some problems of unlikely intersections in arithmetic and geometry, Annals of Mathematics Studies, vol. 181, Princeton University Press, Princeton, NJ, 2012, With appendixes by David Masser.

[Zha95a] S. Zhang, Positive line bundles on arithmetic varieties, J. Amer. Math. Soc. 8 (1995), $187-221$.

[Zha95b] S. Zhang, Small points and adelic metrics, J. Alg. Geom. 4 (1995), 281-300. 
Dragos Ghioca, Department of Mathematics, University of British Columbia, Vancouver, BC V6T 1Z2, CANADA

E-mail address: dghioca@math.ubc.ca

Holly Krieger, Department of Mathematics, Massachusetts Institute of Technology, 77 MasSachusetts Avenue, Cambridge, MA 02139, USA

E-mail address: hkrieger@math.mit.edu

Khoa Nguyen, Department of Mathematics, University of British Columbia, Vancouver, BC V6T 1Z2, CANADA

E-mail address: dknguyen@math.ubc.ca

Hexi Ye, Department of Mathematics, University of British Columbia, Vancouver, BC V6T 1Z2, CANAdA

E-mail address: yehexi@math.ubc.ca 